\title{
A recognition algorithm for adjusted interval digraphs
}

\author{
Asahi Takaoka ${ }^{\mathrm{a}}$ \\ ${ }^{a}$ Department of Information Systems Creation, Faculty of Engineering, Kanagawa University, \\ Rokkakubashi 3-27-1, Kanagawa-ku, Yokohama-shi, Kanagawa, 221-8686, Japan
}

\begin{abstract}
Min orderings give a vertex ordering characterization, common to some graphs and digraphs such as interval graphs, complements of threshold tolerance graphs (known as co-TT graphs), and two-directional orthogonal ray graphs. An adjusted interval digraph is a reflexive digraph that has a min ordering. Adjusted interval digraph can be recognized in $O\left(n^{4}\right)$ time, where $n$ is the number of vertices of the given graph. Finding a more efficient algorithm is posed as an open question. This note provides a new recognition algorithm with running time $O\left(n^{3}\right)$. The algorithm produces a min ordering if the given graph is an adjusted interval digraph.
\end{abstract}

Keywords: Adjusted interval digraphs, Min ordering, Recognition algorithm 2000 MSC: 68R10, 05C75

\section{Introduction}

All graphs and directed graphs (digraphs for short) considered in this paper are finite and have no multiple edges but may have loops. We write $u v$ for the undirected edge joining a vertex $u$ and a vertex $v$; we write $(u, v)$ for the directed edge from $u$ to $v$. We write $V(H)$ for the vertex set of a digraph $H$; we write $E(H)$ for the edge set of $H$. We say that $u$ dominates $v$ (and that $v$ is dominated by $u$ ) in a digraph $H$ if $(u, v) \in E(H)$, and denote it by $u \rightarrow v$ or $v \leftarrow u$.

A digraph $H$ is an interval digraph [5] if for each vertex $v$ of $H$, there is a pair of intervals $I_{v}$ and $J_{v}$ on the real line such that $u \rightarrow v$ in $H$ if and only if $I_{u}$ intersects $J_{v}$. An interval digraph is an adjusted interval digraph [2] if the two intervals $I_{v}$ and $J_{v}$ have the same left endpoint for each vertex $v$. A digraph is called reflexive if every vertex has a loop, and every adjusted interval digraph is reflexive by definition.

Email address: takaoka@kanagawa-u.ac.jp (Asahi Takaoka) 
Adjusted interval digraphs have been introduced by Feder et al. [2] in connection with the study of list homomorphisms. They have shown two characterizations and a recognition algorithm of this graph class.

A min ordering of a digraph $H$ is a linear ordering $\prec$ of the vertices of $H$ such that for any two edges $(u, v)$ and $\left(u^{\prime}, v^{\prime}\right)$ of $H$, we have $\left(u, v^{\prime}\right) \in E(H)$ if $u<u^{\prime}$ and $v^{\prime}<v$. We remark that $(u, v)$ can be a loop, and similarly for $\left(u^{\prime}, v^{\prime}\right)$. A reflexive digraph has a min ordering if and only if it is an adjusted interval digraph [2]. Min orderings give similar characterizations of some graph classes such as interval graphs, complements of threshold tolerance graphs (known as co-TT graphs) [4], two-directional orthogonal ray graphs [6], and signed-interval digraphs [3]. See [3] for details.

Suppose that a digraph $H$ has a min ordering $<$, and let $(u, v),\left(u^{\prime}, v^{\prime}\right)$ be two edges of $H$ with $\left(u, v^{\prime}\right) \notin E(H)$. We have $v \neq v^{\prime}$ from $(u, v) \in E(H)$ and $\left(u, v^{\prime}\right) \notin$ $E(H)$; similarly, we have $u \neq u^{\prime}$ from $\left(u^{\prime}, v^{\prime}\right) \in E(H)$ and $\left(u, v^{\prime}\right) \notin E(H)$. If $u<u^{\prime}$ and $v^{\prime}<v$, then $\left(u, v^{\prime}\right) \in E(H)$ by the property of min orderings, a contradiction. Thus, $u<u^{\prime}$ implies $v<v^{\prime}$ and $v^{\prime}<v$ implies $u^{\prime}<u$. We can capture this forcing relation with an auxiliary digraph. The pair digraph $H^{+}$associated with a digraph $H$ is a digraph such that the vertex set $V\left(H^{+}\right)$is the set $\{(u, v): u \neq v\}$ of ordered pair of two vertices of $H$, and $\left(u, u^{\prime}\right) \rightarrow\left(v, v^{\prime}\right)$ and $\left(v^{\prime}, v\right) \rightarrow\left(u^{\prime}, u\right)$ in $H^{+}$if and only if $(u, v),\left(u^{\prime}, v^{\prime}\right) \in E(H)$ and $\left(u, v^{\prime}\right) \notin E(H)$.

An invertible pair of a digraph $H$ is a pair of two vertices $u, v$ of $H$ such that in $H^{+}$, the vertices $(u, v)$ and $(v, u)$ are in the same strong component. It is clear that if $H$ has an invertible pair, then $H$ does not have any min ordering. Feder et al. [2] have shown that the converse also holds; therefore, a reflexive digraph has no invertible pairs if and only if it has a min ordering.

The characterizations of adjusted interval digraphs yield a recognition algorithm with running time $O\left(m^{2}+n^{2}\right)$, where $n$ and $m$ are the number of vertices and edges of the given graph, respectively [2]. Finding a linear-time recognition algorithm is posed as an open question [2, 3]. In this paper, we show an $O\left(n^{3}\right)$-time recognition algorithm for adjusted interval digraphs. The algorithm produces a min ordering or finds an invertible pair of the given graph if it exists. As a byproduct, we also give an alternative proof to show that a reflexive digraph has a min ordering if and only if it has no invertible pairs.

\section{Algorithm}

In the case of reflexive digraphs, there is an equivalent simpler definition of min orderings. 
Theorem 1 (Feder et al. [2]). Let $H$ be a reflexive digraph. A linear ordering $<$ of the vertices of $H$ is a min ordering if and only if for any three vertices $u, v, w$ with $u<v<w$,

- $(u, w) \in E(H)$ implies $(u, v) \in E(H)$, and

- $(w, u) \in E(H)$ implies $(v, u) \in E(H)$.

In other words, a linear ordering $<$ of the vertices of a reflexive digraph is a min ordering if it contains no triples of vertices $u, v, w$ with $u<v<w$ such that $(u, w) \in E(H)$ and $(u, v) \notin E(H)$, or $(w, u) \in E(H)$ and $(v, u) \notin E(H)$. We call such triples of vertices the forbidden patterns.

Let $H$ be an adjusted interval digraph with a min ordering <. Let $u, v, w$ be distinct vertices of $H$ with $(u, w) \in E(H)$ and $(u, v) \notin E(H)$, or $(w, u) \in E(H)$ and $(v, u) \notin E(H)$. In both cases, if $u<v<w$ then we have a forbidden pattern. Thus, $u<v$ implies $w<v$ and $v<w$ implies $v<u$. To capture this forcing relation, we define an auxiliary digraph associated with $H$.

Definition 2. Let $H$ be a reflexive digraph. The implication graph $H^{*}$ of $H$ is a digraph such that the vertex set $V\left(H^{*}\right)$ is the set $\{(u, v): u \neq v\}$ of ordered pair of two vertices of $H$, and for any three vertices $u, v, w$ of $H$, we have $(u, v) \rightarrow(w, v)$ and $(v, w) \rightarrow(v, u)$ in $H^{*}$ if and only if

- $(u, w) \in E(H)$ and $(u, v) \notin E(H)$, or

- $(w, u) \in E(H)$ and $(v, u) \notin E(H)$.

We can use the implication graphs for recognizing adjusted interval digraphs.

Lemma 3. Let $H$ and $H^{*}$ be a reflexive digraph and its implication graph, respectively. A pair of two vertices $u, v \in V(H)$ is an invertible pair if and only if in $H^{*}$, the vertices $(u, v)$ and $(v, u)$ are in the same strong component.

Proof. Let $H^{+}$be the pair digraph of $H$. Let $u, v, w$ be three vertices of $H$ such that $(u, v) \rightarrow(w, v)$ in $H^{*}$ (or equivalently, $(v, w) \rightarrow(v, u)$ in $\left.H^{*}\right)$. By definition, $(u, w) \in E(H)$ and $(u, v) \notin E(H)$, or $(w, u) \in E(H)$ and $(v, u) \notin E(H)$. Since the vertex $v$ has a loop, in both cases $(u, v) \rightarrow(w, v)$ and $(v, w) \rightarrow(v, u)$ in $H^{+}$. Therefore, $H^{*}$ is a subgraph of $H^{+}$.

Assume that $(u, v) \rightarrow\left(u^{\prime}, v^{\prime}\right)$ in $H^{+}$. By definition, $\left(u, u^{\prime}\right),\left(v, v^{\prime}\right) \in E(H)$ and $\left(u, v^{\prime}\right) \notin E(H)$, or $\left(u^{\prime}, u\right),\left(v^{\prime}, v\right) \in E(H)$ and $\left(v^{\prime}, u\right) \notin E(H)$. In both cases, if $\left(u, u^{\prime}\right)$ or $\left(v, v^{\prime}\right)$ is a loop, then $(u, v) \rightarrow\left(u^{\prime}, v^{\prime}\right)$ in $H^{*}$. Thus we may assume $u \neq u^{\prime}$ and $v \neq v^{\prime}$. We have $u \neq v^{\prime}$ since $H$ is reflexive. Recall that $u \neq v$ and $u^{\prime} \neq v^{\prime}$. Thus, 
the vertices $u, v, v^{\prime}$ are distinct, and $(u, v) \rightarrow\left(u, v^{\prime}\right)$ in $H^{*}$. Similarly, the vertices $u, u^{\prime}, v^{\prime}$ are distinct, and $\left(u, v^{\prime}\right) \rightarrow\left(u^{\prime}, v^{\prime}\right)$ in $H^{*}$. Therefore, if $(u, v) \rightarrow\left(u^{\prime}, v^{\prime}\right)$ in $H^{+}$, then $H^{*}$ has a directed path from $(u, v)$ to $\left(u^{\prime}, v^{\prime}\right)$.

Lemma 3 gives an algorithm to find an invertible pair if it exists. Given a reflexive digraph $H$, the algorithm first construct the implication graph $H^{*}$ of $H$, then compute the strong components of $H^{*}$, and finally check for the existence of a pair $(u, v)$ and $(v, u)$ within one strong component. The implication graph $H^{*}$ has $n(n-1)$ vertices, and at most $2 n m$ edges since $H^{*}$ has at most two edges for each pair of a vertex and an edge of $H$. Therefore, we can construct $H^{*}$ in time $O(n m)$, and check for the existence of invertible pairs in the same time bound.

We next describe the algorithm for producing a min ordering of an adjusted interval digraph. Let $H$ and $H^{*}$ be a reflexive digraph and its implication graph, respectively. As an auxiliary graph, we use a complete graph $K$ with the vertex set $V(H)$. An orientation of $K$ is a digraph obtained from $K$ by orienting each edge of $K$, that is, replacing each edge $u v \in E(K)$ with either $(u, v)$ or $(v, u)$. An orientation of $K$ is acyclic if it contains no directed cycles; an acyclic orientation of $K$ is equivalent to a linear ordering of the vertices of $H$.

We say that a vertex $(u, v)$ of $H^{*}$ is an implicant of a vertex $\left(u^{\prime}, v^{\prime}\right)$ if $H^{*}$ has a directed walk from $\left(u^{\prime}, v^{\prime}\right)$ to $(u, v)$. We say that an orientation $T$ of $K$ is consistent with $H$ if for each vertex $(u, v)$ of $H^{*}$, we have $u \rightarrow v$ in $T$ implies $u^{\prime} \rightarrow v^{\prime}$ for every implicant $\left(u^{\prime}, v^{\prime}\right)$ of $(u, v)$. It is clear that an acyclic orientation of $K$ is consistent with $H$ if and only if it contains no forbidden patterns of min orderings. Therefore, a min ordering of $H$ is equivalent to an orientation of $K$ that is acyclic and consistent with $H$.

It is sufficient for the existence of a min ordering of $H$ that there is an orientation of $K$ consistent with $H$.

Lemma 4. There is a min ordering of $H$ if and only if there is an orientation of $K$ consistent with $\mathrm{H}$.

Let $T$ be an orientation of $K$ consistent with $H$ that is not acyclic. In order to prove Lemma 4, we provide an algorithm for producing another orientation of $K$ that is acyclic and consistent with $H$.

A directed triangle is a directed cycle of length 3. It is well known that an orientation of a complete graph is acyclic if and only if it contains no directed triangles. Let $u$ be a vertex of $K$, and let $E_{u}$ be the set of all the edges $(v, w) \in E(T)$ such that $u \rightarrow v, v \rightarrow w$, and $w \rightarrow u$ in $T$. The reversal $E_{u}^{-}$of $E_{u}$ is the set of directed edges obtained from $E_{u}$ by reversing the direction of all the edges 
in $E_{u}$, that is, $E_{u}^{-}=\left\{(x, y):(y, x) \in E_{u}\right\}$. We define that $T^{\prime}$ is the orientation of $K$ obtained from $T$ by reversing the direction of all the edges in $E_{u}$, that is, $E\left(T^{\prime}\right)=\left(E(T)-E_{u}\right) \cup E_{u}^{-}$.

We will show that the orientation $T^{\prime}$ has the following properties: $T^{\prime}$ is still consistent with $H ; T^{\prime}$ contains no directed triangles having the vertex $u$; the reversing the direction of edges in $E_{u}$ generates no directed triangles. Therefore, by repeated application of this procedure for each vertex of $K$, we can obtain an orientation of $K$ that is acyclic and consistent with $H$; the complexity of the algorithm is $O\left(n^{3}\right)$.

To show that $T^{\prime}$ is still consistent with $H$, we prove a lemma for directed triangles in the orientation of $K$ consistent with $H$.

Lemma 5. Let $T$ be an orientation of $K$ consistent with $H$. Suppose that $T$ has three vertices $u, v, w$ such that $u \rightarrow v, v \rightarrow w$, and $w \rightarrow u$ in $T$. If $v^{\prime} \rightarrow w^{\prime}$ in $T$ and $\left(v^{\prime}, w^{\prime}\right) \rightarrow(v, w)$ in $H^{*}$, then $u \rightarrow v^{\prime}, v^{\prime} \rightarrow w^{\prime}$, and $w^{\prime} \rightarrow u$ in $T$.

Proof. We say that a set of vertices $S \subseteq V(H)$ is complete if $(x, y),(y, x) \in E(H)$ for any two vertices $x, y \in S$, and is independent if $(x, y),(y, x) \notin E(H)$. We claim that the set of vertices $\{u, v, w\}$ is complete or independent. Suppose $(u, v) \in E(H)$. If $(w, v) \notin E(H)$, then $(v, w) \rightarrow(u, w)$ in $H^{*}$, a contradiction. Thus $(w, v) \in E(H)$. If $(w, u) \notin E(H)$, then $(w, u) \rightarrow(v, u)$ in $H^{*}$, a contradiction. Thus $(w, u) \in E(H)$. If $(v, u) \notin E(H)$, then $(u, v) \rightarrow(w, v)$ in $H^{*}$, a contradiction. Thus $(v, u) \in E(H)$. Continuing in this way, we have that $\{u, v, w\}$ is complete.

We have either $v^{\prime}=v$ or $w^{\prime}=w$. Suppose $v^{\prime}=v$. Since $\left(v^{\prime}, w^{\prime}\right) \rightarrow(v, w)$ in $H^{*}$, we have $(v, w) \notin E(H)$ and $\left(w^{\prime}, w\right) \in E(H)$, or $(w, v) \notin E(H)$ and $\left(w, w^{\prime}\right) \in E(H)$. In both cases, the set of vertices $\{u, v, w\}$ is independent. Since $(u, w) \notin E(H)$ and $\left(w^{\prime}, w\right) \in E(H)$, or $(w, u) \notin E(H)$ and $\left(w, w^{\prime}\right) \in E(H)$, we have $(w, u) \rightarrow\left(w^{\prime}, u\right)$ in $H^{*}$; therefore, $w^{\prime} \rightarrow u$ in $T$.

We next suppose $w^{\prime}=w$. Since $\left(v^{\prime}, w^{\prime}\right) \rightarrow(v, w)$ in $H^{*}$, we have $\left(v^{\prime}, w^{\prime}\right) \notin$ $E(H)$ and $\left(v^{\prime}, v\right) \in E(H)$, or $\left(w^{\prime}, v^{\prime}\right) \notin E(H)$ and $\left(v, v^{\prime}\right) \in E(H)$. Due to symmetry, we may assume $\left(v^{\prime}, w^{\prime}\right) \notin E(H)$ and $\left(v^{\prime}, u\right) \in E(H)$. If $\left(v^{\prime}, u\right) \in E(H)$, we have $\left(v^{\prime}, w^{\prime}\right) \rightarrow(u, w)$ in $H^{*}$, a contradiction. Thus $\left(v^{\prime}, u\right) \notin E(H)$. We now have $(u, v) \rightarrow\left(u, v^{\prime}\right)$ in $H^{*}$, and therefore, $u \rightarrow v^{\prime}$ in $T$.

Suppose that $T^{\prime}$ is not consistent with $H$. Then, there exist three vertices $x, y, z$ such that $x \rightarrow y$ and $y \rightarrow z$ in $T^{\prime}$ but $(x, y) \rightarrow(z, y)$ in $H^{*}$ (or equivalently, $(y, z) \rightarrow(y, x)$ in $\left.H^{*}\right)$. Since $T$ is consistent with $H$, we have $(x, y) \in E_{u}^{-}$or $(y, z) \in E_{u}^{-}$. Suppose $(x, y),(y, z) \in E_{u}^{-}$. We have that $(x, y) \in E_{u}^{-}$implies $u \rightarrow y$ in $T$ and $(y, z) \in E_{u}^{-}$implies $y \rightarrow u$ in $T$, a contradiction. If $(x, y) \in E_{u}^{-}$and 
$(y, z) \notin E_{u}^{-}$, then $(y, x) \in E_{u}$ and $y \rightarrow z$ in $T$. Since $(y, z) \rightarrow(y, x)$ in $H^{*}$, we have from Lemma 5 that $(y, z) \in E_{u}$, a contradiction. Similarly, if $(x, y) \notin E_{u}^{-}$and $(y, z) \in E_{u}^{-}$, then $x \rightarrow y$ in $T$ and $(z, y) \in E_{u}$. Since $(x, y) \rightarrow(z, y)$ in $H^{*}$, we have from Lemma 5 that $(x, y) \in E_{u}$, a contradiction. Therefore, $T^{\prime}$ is still consistent with $H$.

Trivially, $T^{\prime}$ contains no directed triangles having the vertex $u$.

Let $x, y, z$ be three vertices such that $x \rightarrow y, y \rightarrow z$, and $z \rightarrow x$ in $T^{\prime}$. Suppose $(x, y),(y, z) \in E_{u}^{-}$. We have that $(x, y) \in E_{u}^{-}$implies $u \rightarrow y$ in $T$ and $(y, z) \in E_{u}^{-}$ implies $y \rightarrow u$ in $T$, a contradiction. Thus at most one edge on the directed triangle is in $E_{u}^{-}$. Suppose $(x, y) \in E_{u}^{-}$and $(y, z),(z, x) \notin E_{u}^{-}$. We have $u \rightarrow y, y \rightarrow z, z \rightarrow x$, and $x \rightarrow u$ in $T$. If $u \rightarrow z$ in $T$ then $(z, x) \in E_{u}$; if $z \rightarrow u$ in $T$ then $(y, z) \in E_{u}$, a contradiction. Therefore, the reversing the direction of edges in $E_{u}$ generates no directed triangles, and we have Lemma 4.

We now show an algorithm for finding an orientation of $K$ consistent with $H$. We use an algorithm for the 2-satisfiability problem. An instance of the 2satisfiability problem is a $2 C N F$ formula, a Boolean formula in conjunctive normal form with at most two literals per clause. We construct the $2 \mathrm{CNF}$ formula $\phi_{H}$ associated with $H$. Assume that the vertices of $H$ are linearly ordered, and let $x_{(u, v)}$ be a Boolean variable if a vertex $u$ of $H$ precedes a vertex $v$ in this ordering. We denote the negation of $x_{(u, v)}$ by $x_{(v, u)}$. We define that $\phi_{H}$ is a $2 \mathrm{CNF}$ formula consisting of all the clauses $\left(x_{(u, v)} \vee x_{(v, w)}\right)$ such that $(u, w) \in E(H)$ and $(u, v) \notin$ $E(H)$, or $(w, u) \in E(H)$ and $(v, u) \notin E(H)$.

Let $\tau$ be a truth assignment of $\phi_{H}$. We define that an orientation of $K$ associated with $\tau$ is an orientation $T_{\tau}$ such that $u \rightarrow v$ in $T_{\tau}$ if and only if $x_{(u, v)}=0$ in $\tau$ for any two vertices $u, v$ of $K$. It is clear from the construction of $\phi_{H}$ that $T_{\tau}$ is consistent with $H$ if and only if $\tau$ satisfies $\phi_{H}$.

Lemma 6. There is an orientation of $K$ consistent with $H$ if and only if $\phi_{H}$ is satisfiable.

The 2CNF formula $\phi_{H}$ has at most $n(n-1) / 2$ Boolean variables, and at most $n m$ clauses since $\phi_{H}$ has at most one clause for each pair of a vertex and an edge of $H$. Thus $\phi_{H}$ can be constructed in $O(n m)$ time. Since a satisfying truth assignment of $\phi_{H}$ can be computed in time linear to the size of $\phi_{H}$ (see [1] for example), we can find an orientation of $K$ consistent with $H$ in $O(n m)$ time.

Let $\phi$ be a 2CNF formula. For a Boolean variable $x_{i}$ in $\phi$, the negation of $x_{i}$ is denoted by $\overline{x_{i}}$. The implication graph $G(\phi)$ of $\phi$ is the digraph constructed as follows: for each variable $x_{i}$, we add two vertices named $x_{i}$ and $\overline{x_{i}}$ to $G(\phi)$; for each clause $\left(x_{i}, x_{j}\right)$, we add two edges to $G(\phi)$ so that $\overline{x_{i}} \rightarrow x_{j}$ and $\overline{x_{j}} \rightarrow x_{i}$. A 
$2 \mathrm{CNF}$ formula $\phi$ is satisfiable if and only if in $G(\phi)$, any pair of vertices $x_{i}$ and $\overline{x_{i}}$ are not in the same strong component [1].

For a reflexive digraph $H$, it is clear from the construction of $\phi_{H}$ and $H^{*}$ that $G\left(\phi_{H}\right)$ is isomorphic to the subgraph of $H^{*}$ obtained by removing all the isolated vertices of $H^{*}$. Therefore, we have the following.

Lemma 7. The 2 CNF formula $\phi_{H}$ is satisfiable if and only if $H$ has no invertible pairs.

From Lemmas 4, 6, and 7, we now have an alternative proof for the theorem of Feder et al. [2].

Theorem 8. A reflexive digraph has a min ordering if and only if it contains no invertible pairs.

We finally summarize our algorithm for recognizing adjusted interval graphs. This algorithm produces a min ordering of the given graph if it is an adjusted interval digraph, and finds an invertible pair if otherwise.

Algorithm 9. Let $H$ be a reflexive digraph.

Step 1. Compute a 2CNF formula $\phi_{H}$ from $H$.

Step 2. Find a satisfying truth assignment of $\phi_{H}$.

If $\phi_{H}$ is satisfiable, go to Step 3. Otherwise, go to Step 4.

Step 3. Let $\tau$ be a satisfying truth assignment of $\phi_{H}$.

Compute an orientation $T_{\tau}$ of $K$ associated with $\tau$.

Compute a min ordering of $H$ from $T_{\tau}$ if $T_{\tau}$ is not acyclic.

Output the min ordering of $H$, and halt.

Step 4. Construct the implication graph $H^{*}$ of $H$. Then, find an invertible pair. Output the invertible pair of $H$, and halt.

The correctness of the algorithm follows from Lemmas 4, 6, and 7, Steps 1, 2, and 4 can be performed in $O(n m)$ time; Step 3 can be performed in $O\left(n^{3}\right)$ time.

Theorem 10. Adjusted interval digraphs can be recognized in $O\left(n^{3}\right)$ time.

\section{References}

[1] B. Aspvall, M. F. Plass, and R. E. Tarjan. A linear-time algorithm for testing the truth of certain quantified boolean formulas. Inf. Process. Lett., 8(3):121-123, 1979. 
[2] T. Feder, P. Hell, J. Huang, and A. Rafiey. Interval graphs, adjusted interval digraphs, and reflexive list homomorphisms. Discrete Appl. Math., 160(6):697-707, 2012.

[3] P. Hell, J. Huang, R. M. McConnell, and A. Rafiey. Interval-Like Graphs and Digraphs. In I. Potapov, P. Spirakis, and J. Worrell, editors, Proceedings of the 43rd International Symposium on Mathematical Foundations of Computer Science (MFCS 2018), volume 117 of Leibniz International Proceedings in Informatics (LIPIcs), pages 68:1-68:13. Schloss Dagstuhl - Leibniz-Zentrum für Informatik, 2018.

[4] C. L. Monma, B. Reed, and W. T. Trotter. Threshold tolerance graphs, $J$. Graph Theory, 12(3):343-362, 1988.

[5] M. Sen, S. Das, A. B. Roy, and D. B. West. Interval digraphs: An analogue of interval graphs. $\quad J$. Graph Theory, 13(2):189-202, 1989.

[6] A. M. S. Shrestha, S. Tayu, and S. Ueno. On orthogonal ray graphs. Discrete Appl. Math., 158(15):1650-1659, 2010. 